\title{
La transparencia sobre sostenibilidad en gobiernos regionales: el caso de España
}

\author{
Transparency about sustainability in regional governments: \\ the case of Spain
}

Francisco J. Alcaraz-Quiles / falcaraz@ugr.es
Andrés Navarro-Galera / angalera@ugr.es
David Ortiz-Rodríguez / dortiz@ugr.es
Universidad de Granada,España

\begin{abstract}
In the current crisis, citizens are demanding greater public sector transparency and accountability. In this paper, we advance the understanding of sustainability practices in public administration. We propose to compare the dissemination of sustainability of various regional governments, analyzing the websites of these regional governments through a content analysis, using for this purpose a list of items obtained from the Global Reporting Initiative (GRI) guidelines.

Our results show that Spanish regional governments, spread two-thirds of the information in the GRI guidelines, being the social information which presents a higher level of response and corresponding general aspects which has a lower level of dissemination. In addition, those regional governments with greater environmental commitment are those who publish more information about sustainability. From our conclusions, we emphasize that the poor financial situation of the Spanish regional governments does not seem to promote them to disseminate information about sustainability.
\end{abstract}

Key words: sustainability, Corporate Social Responsibility (CSR), regional governments, dissemination of information.

Resumen: En el actual contexto de crisis, los ciudadanos demandan al sector público mayor transparencia y rendición de cuentas. Este trabajo pretende avanzar en el conocimiento de las prácticas de sostenibilidad en la administración pública. Para ello proponemos comparar la divulgación de información sobre sostenibilidad realizada por distintos gobiernos regionales, analizando las webs de los mismos mediante un análisis de contenido, usando para ello un listado de ítems basado en las guías del Global Reporting Initiative (GRI).

Nuestros resultados muestran que los gobiernos regionales españoles difunden dos tercios de la información requerida por el GRI, presentando la información social un nivel de respuesta mayor y la información general un menor nivel de difusión. Además, aquellos gobiernos regionales con mayor compromiso ambiental publican más información sobre sostenibilidad. De nuestras conclusiones podemos destacar que la mala situación financiera que atraviesan no parece impulsar a los gobiernos regionales españoles a divulgar información sobre sostenibilidad.

Palabras clave: sostenibilidad, Responsabilidad Social Corporativa (RSC), gobiernos regionales, divulgación de información. 


\section{Introducción ${ }^{1}$}

La crisis económica y los escándalos financieros han provocado que los ciudadanos reclamen mayor transparencia y compromiso por parte de las entidades empresariales. Internacionalmente, se ha venido produciendo un paulatino crecimiento de la preocupación acerca de los impactos sociales y medioambientales de las organizaciones (Elkington, 1998; Osborne y Ball, 2011). Así, la sostenibilidad, en su triple dimensión de viabilidad económica, social y medioambiental, se ha convertido en un concepto central en el contexto económico (International Federation of Accountants [IFAC], 2011).

En respuesta a la sensibilidad ciudadana sobre estos temas, las grandes empresas han incrementado, como complemento a los datos económicos y financieros publicados tradicionalmente, la cantidad de información social y medioambiental que emiten (Gray, 2006). Por tanto, actualmente los informes de sostenibilidad (IS) representan un concepto esencial en el mundo empresarial, donde han sido ampliamente estudiados (Banerjee, 2007).

En el ámbito público, la divulgación de información sobre sostenibilidad está siendo uno de los aspectos más innovadores en la relación de los gobiernos con los ciudadanos, por dos motivos. En primer lugar, organismos internacionales como la Unión Europea (UE), la Organización para la Cooperación y el Desarrollo Económicos (OCDE), Naciones Unidas (NU) y el Banco Mundial (BM) han recomendado a los gobiernos la adopción de prácticas socialmente responsables en sus políticas, así como la emisión de información hacia los ciudadanos acerca de la sostenibilidad de sus acciones en materia social, económica y medioambiental (UE, 2007 y 2011; IFAC, 2013; Grupo Banco Mundial, 2007; OCDE, 2006).

El segundo de los motivos está relacionado con las demandas recibidas por las entidades del ámbito público. Diversos stakeholders (agentes afectados directa o indirectamente por las actuaciones) están solicitando que las acciones llevadas a cabo por las administraciones públicas sean cada vez más sostenibles, y que aumente la información sobre la sostenibilidad gubernamental (Coglianese, 2009; Crane et al., 2008). En este sentido, la transparencia y la rendición de cuentas son los dos pilares principales sobre los que el gobierno debe construir el sector público, ofreciendo los informes de sostenibilidad

1 Agradecimientos: Esta investigación fue realizada con la financiación de la Comunidad Autónoma de Andalucía, Consejería de Innovación, Ciencia y Empresa (Proyecto de Investigación núm. P09-SEJ-5395), así como del Ministerio de Ciencia e Innovación (Proyecto de Investigación núm. ECO2010-17463-ECON-FEDER y Proyecto de Investigación núm. ECO2010-20522-ECON-FEDER). 
mecanismos para reflexionar sobre lo que quieren lograr y motivaciones para sus acciones futuras (Bebbington et al., 2008; G-20, 2013; IFAC, 2013).

Aunque el sector público ha actuado tradicionalmente como promotor para la incorporación de prácticas socialmente responsables (Crane y Matten, 2007), los stakeholders están demandando que las administraciones públicas actúen de forma socialmente responsable (Crane et al., 2008) y que se produzca una adecuada difusión y control a través de memorias de sostenibilidad o auditorías sociales (Ball, 2004), pues, en comparación con el ámbito privado, todavía son pocas las entidades públicas que emiten informes sobre sostenibilidad (Frost, 2007; Mussari y Monfardini, 2010) y son escasos los estudios académicos al respecto (Osborne y Ball, 2011). Este es el motivo, como señala Contreras (2010), para que los gobiernos se hayan visto en la obligación de iniciar procesos de modernización y reformas en el aparato público, a fin de poder adaptarse a las características del medio ambiente cambiante.

Así pues, nuestro objetivo es observar la prácticas de difusión de información sobre sostenibilidad en el ámbito público y qué tipo de información está siendo difundida. Además, en una primera aproximación, pretendemos observar cuál es la influencia que algunos factores (déficit, deuda pública o nivel general de transparencia de la entidad) pueden tener en la divulgación de este tipo de información por parte de las entidades públicas.

En orden a estos objetivos, creemos interesante enfocar nuestro estudio en los Gobiernos Regionales españoles, denominados, según el ordenamiento jurídico constitucional español, como Comunidades Autónomas (CCAA en adelante), por varios motivos. Como apunta Potts (2010), existe un interés creciente sobre la sostenibilidad de las CCAA. Ello es así porque, en el contexto de crisis de las finanzas públicas y el cuestionamiento sobre la existencia de los distintos niveles del sector público, las CCAA, desde su situación intermedia entre la administración central y local, están obligados a afrontar los retos actuales sobre comunicación y desarrollo sostenible (Lansu et al., 2013).

En este sentido, creemos que las CCAA españolas son un adecuado ámbito de estudio puesto que, en el marco de un elevado nivel de déficit (18.000 millones de euros) (IGAE, 2015) y creciente deuda pública (Eurostat, 2012), su funcionamiento y actuaciones están siendo muy cuestionadas, motivo por el cual, según distintas teorías, en particular la Teoría de la Legitimación y la de los Stakeholder, sus responsables deberían estar especialmente interesados en mostrar a los ciudadanos la sostenibilidad de sus actuaciones, ya sea para legitimarse o para responder a las demandas ciudadanas. 
El resto del trabajo se organiza como sigue: en el apartado segundo presentamos una revisión del concepto de responsabilidad social y la emisión de informes de sostenibilidad en entidades públicas. En el apartado tercero describimos tanto la muestra como la metodología del estudio, mientras en el cuarto exponemos los principales resultados de nuestra observación. Finalizamos recogiendo algunas conclusiones y reflexiones.

\section{Responsabilidad social e informes de sostenibilidad en administraciones públicas}

El sector público desempeña un doble papel respecto de la sostenibilidad. Por una parte, como señalan Fox et al. (2002), las entidades públicas tienen que obligar, facilitar, colaborar y promocionar la introducción de prácticas de sostenibilidad. Por otra parte, según Erdmenger (1998), las administraciones públicas no sólo deben implementar políticas que incentiven el desarrollo sostenible, sino también realizar una administración interna acorde con estas prácticas.

En esta línea, como señala Uvalle-Berrones (2012), la administración pública en la sociedad contemporánea se transforma a fin de potenciar la democracia y eficiencia, con lo cual su esquema de organización, gestión y comportamiento tiene ahora referentes que la ubican en el espacio público con nuevas exigencias relacionadas con la calidad de su desempeño, la efectividad de la transparencia y la importancia de la rendición de cuentas.

Para autores como Lamprinidi y Kubo (2008), las razones por las que las entidades públicas deberían estar interesadas en informar sobre sostenibilidad no difieren de las que tienen las entidades privadas: 1) necesidad de demostrar esfuerzos sobre el cambio climático y otras cuestiones de sostenibilidad; 2) un creciente interés y demanda en transparencia y rendición de cuentas; 3) explicación de la gestión realizada a los stakeholders; 4) mostrar liderazgo en el sector; 5) existencia de "campeones" de informes de sostenibilidad que quieren promover la presentación de informes de sostenibilidad y compartir los beneficios que han experimentado; 6) el informe de sostenibilidad puede ser construido a partir de requisitos de la información existentes.

En un contexto como el actual, marcado por la crisis de las finanzas públicas y la creciente demanda de mejores servicios públicos por parte de los ciudadanos, la justificación teórica para la puesta en marcha de prácticas de sostenibilidad en el sector público también puede ser diversa, si bien los enfoques más utilizados son la Teoría de la Legitimidad (TL) (Neu et al., 1998) y la Teoría de los Stakeholder (TS) (Deegan y Unerman, 2006). 
La legitimidad está considerada como el logro de la armonización entre el comportamiento de las organizaciones y los valores de la sociedad. La TL describe cómo las organizaciones implantan medidas tendentes a reducir la brecha entre los posibles perjuicios para la ciudadanía por las actividades llevadas a cabo por aquellas, y los valores prevalentes en la sociedad civil (Lodhia et al., 2012). La legitimidad es un recurso del cual las organizaciones dependen para sobrevivir, y en el momento en que los gestores consideran que el suministro de determinado elemento es esencial para la supervivencia, desarrollan estrategias para asegurar la continuidad del suministro del elemento en cuestión (Deegan, 2002).

La TS sostiene que el gobierno de las organizaciones ha de considerar no sólo los objetivos más directos y concretos, sino también el contexto, dando respuesta a las demandas de las múltiples partes interesadas (stakeholders) (Marcuccio y Steccolini, 2005); por lo que los responsables públicos deberían satisfacer las demandas de todos los grupos interesados, teniendo en cuenta sus intereses y expectativas (Goodpaster, 2009).

Sin embargo, independientemente del enfoque teórico utilizado para justificar los motivos por los que la información sobre sostenibilidad es divulgada, en la actualidad la sostenibilidad ha llegado a ser considerada universalmente como un elemento clave en las organizaciones del Sector Público (Guthrie et al., 2010).

En cualquier caso, y a nuestro juicio, la publicación de información sobre sostenibilidad, además de suponer un incremento en la transparencia, es beneficiosa para las entidades públicas, puesto que, tal como evidencian Bebbington et al. (2008), informar sobre sostenibilidad proporciona a las organizaciones mecanismos para reflexionar sobre lo que pretenden alcanzar, motivando así acciones futuras.

En este contexto, numerosos organismos internacionales (como OCDE, NU, BM, IFAC o la UE) están recomendando a los gobiernos que adopten prácticas de responsabilidad social, informando al ciudadano sobre la sostenibilidad de sus actuaciones en materia social, económica y medioambiental (UE, 2007, 2011, 2013; IFAC, 2013; Grupo del Banco Mundial, 2007; OCDE, 2006).

De acuerdo con ello, el creciente interés en los informes sobre sostenibilidad ha supuesto la publicación de diferentes guías para difundir información sobre prácticas de sostenibilidad, que pueden ser de utilidad para las entidades públicas (Martinov-Bennie y Hecimovic, 2010). Entre otras, podemos mencionar las emitidas por la OCDE (2006), el Banco Mundial (2008), AccountAbility (2008a y 2008b) o el Pacto Mundial de Naciones Unidas 
(2009), pero la propuesta del Global Reporting Initiative (GRI), cuya versión más reciente es conocida como G3 (GRI, 2006), es considerada internacionalmente como el prototipo de guía para elaborar informes de sostenibilidad (Crognale, 2009).

En este sentido, como evidencian Lodhia et al. (2012), las guías del GRI se han convertido en la referencia para la publicación de informes de sostenibilidad a nivel internacional, incorporando indicadores para medir la gestión en cuestiones económicas, sociales y medioambientales.

Entre los suplementos de sector (propuestas complementarias elaboradas para ámbitos concretos), el GRI publicó un documento piloto dedicado a entidades públicas (GRI, 2005). Después de un análisis del mismo y de su uso (Tort, 2010), el GRI ha publicado en 2010 un nuevo suplemento específico (GRI, 2010).

El número de informes que se emiten por entidades públicas aplicando las recomendaciones de las guías del GRI está creciendo de forma continuada (Dumay et al., 2010), si bien se está usando más la guía general que el suplemento específico para entidades públicas (Tort, 2010). Además, se puede afirmar que, en el ámbito público, las guías GRI han tenido un gran impacto (Canyelles, 2011).

Los estudios sobre la difusión de información de sostenibilidad en entidades públicas, como señalan Lodhia et al. (2012), son aún escasos. Entre los publicados podemos mencionar los de Herbohn (2005), Farneti y Guthrie (2009), Mussari y Monfardini (2010), Navarro-Galera et al. (2010) o Lodhia et al. (2012). Por ello, como apuntan Guthrie et al. (2010), son necesarios más estudios sobre la sostenibilidad y la rendición de cuentas en entidades públicas. De este modo, como señalan Marcuccio y Steccolini (2005), existen dos líneas interesantes de investigación: el análisis de los sistemas de información sobre sostenibilidad y el estudio de por qué la información debería ser publicada.

Por tanto, a la vista de, por un lado, la situación de crisis financiera de las entidades públicas, que hace precisa, además de una mejora en la gestión, un incremento de la transparencia hacia los ciudadanos y, por otro, el reducido número de estudios sobre difusión de información de sostenibilidad por parte de las entidades públicas, creemos interesante y oportuno realizar un estudio al respecto en el ámbito de los gobiernos regionales. Con ello podremos tener una mejor idea de cómo, en el ámbito de la divulgación de información de sostenibilidad, están actuando los gobiernos descentralizados, al tiempo que observaremos el efecto que, en este sentido, tienen el déficit, la deuda y la demanda de transparencia. 


\section{Selección de la muestra y metodología}

\section{Selección de la muestra}

En este trabajo nos centramos en los gobiernos regionales españoles. La selección de las CCAA está justificada por el hecho, como señalan Jordana et al. (2005), de que el estudio comparativo entre regiones de un mismo país puede presentar ventajas frente a estudios entre países. Ello es así porque las regiones tienen diferencias significativas en lo político, económico y social, pero otras variables con efectos sobre la difusión de información, como por ejemplo la legislación, aquí permanecen constantes, lo cual favorece el análisis de las observaciones.

Una vez centrado el estudio en las CCAA, creemos que el caso español es particularmente interesante por dos motivos directamente relacionados con los procesos de legitimación y transparencia gubernamental. El primero, la difícil situación financiera por la que están atravesando las entidades públicas españolas. El segundo, el rápido y profundo proceso de descentralización realizado en España, que ha supuesto que un considerable número de competencias y recursos pasen a ser gestionados por las CCAA.

En este sentido, el Estado español es uno de los que más está sufriendo la crisis de las finanzas públicas (Eurostat, 2012), presentando elevados niveles de déficit y deuda pública. Así, a pesar de las importantes medidas de austeridad aplicadas en los últimos años, el nivel de déficit del Sector Público español, sin considerar las ayudas al sector financiero, se ubica como uno de los más altos de Europa, alcanzando casi $9 \%$ del PIB en 2011 y situándose en el 6,74\% del PIB en 2012.

Esta situación ha supuesto la aplicación de recortes en prestaciones públicas junto a incrementos en los niveles impositivos, lo cual ha motivado, aparte del rechazo manifestado por parte de la sociedad, que el conjunto de la ciudadanía reclame mayor transparencia en la gestión pública, tal como ya están realizando algunos gobiernos que están aplicando medidas que inciden en los principios de transparencia y participación.

En el entorno de crisis financiera y de las finanzas públicas, según datos oficiales (IGAE, 2015), las CCAA españolas tienen un déficit de 18.000 millones de euros (200 millones menos que en el ejercicio anterior), un 1.66\% sobre el PIB ( $0.9 \%$ inferior al ratio alcanzado el último año), alcanzando un 36.3\% del gasto público no financiero para 2015 en España. De hecho, la literatura previa y los organismos internacionales reconocen que la existencia continuada de deuda y déficit en las administraciones públicas supone un 
riesgo para la sostenibilidad, por lo que abogan por el establecimiento de medidas de control de ambas magnitudes (Pina et al., 2010; United States Agency for International Development [USAID], 2011; UE, 2013). Esto hace que, en la actualidad, resulte interesante estudiar empíricamente si los niveles de deuda y déficit tienen alguna influencia en la transparencia informativa sobre sostenibilidad.

En cuanto a la descentralización en España, cabe señalar que un aspecto fundamental del proceso constitucional español de finales de la década de 1970 fue la creación de 17 CCAA, con sus correspondientes gobiernos y parlamentos (Muñoz y Chain, 2004). En apenas un cuarto de siglo, el nivel de descentralización alcanzó niveles superiores a los existentes en otros países europeos (Jeffery, 1997; Le Galès y Lequesne, 1998) y, según datos oficiales (IGAE, 2012), las CCAA gestionan más de un tercio del gasto no financiero en España. De este modo, la estructura política y las instituciones de estas CCAA son muy parecidas a las de un sistema federal, de forma que las CCAA, dentro de un marco común, son responsables de un amplio conjunto de políticas, entre otras, educación, salud, empleo, servicios sociales o medioambiente.

Entre los objetivos del proceso de descentralización destacan el impulso del desarrollo sostenible de las regiones, la mayor proximidad al ciudadano y el fomento de la transparencia (Gil-Ruiz e Iglesias, 2007). Sin embargo, en el actual contexto de crisis económica, las CCAA están sufriendo una importante presión para atender las crecientes necesidades derivadas de sus competencias, pues desde 2007 presentan un considerable aumento de sus niveles de deuda, duplicando en este periodo, en términos de PIB nacional, su volumen (Ruiz-Huerta y García, 2012). Así, en el marco de la teoría de la legitimidad, las CCAA están obligadas a analizar sus actuaciones y aplicar medidas de transparencia, dando respuesta, como plantea la teoría de los stakeholders, a los distintos grupos de interés.

Por todo ello, la elección de las CCAA españolas puede ser interesante para los objetivos del estudio por varios motivos. Por un lado, nos encontramos con un conjunto de gobiernos diversos que desarrollan sus políticas de forma autónoma en contextos socioeconómicos diferentes. En particular, las CCAA españolas se enfrentan a una coyuntura compleja, en la cual se conjugan la limitación de recursos, un importante número de competencias que atender y la necesidad de justificar sus actuaciones ante la ciudadanía.

Por otro lado, todas las CCAA tienen un marco legal común, en el que, si bien no existe la obligación de elaborar informes de sostenibilidad, la regulación contable insiste en la inclusión de cierta información social y 
medioambiental en las cuentas anuales (Orden EHA/1037/2010). Por ende, la difusión de información dependerá del interés de los gestores y políticos; por lo tanto, su análisis permitirá conocer qué aspectos de los informes de sostenibilidad son considerados de interés para su difusión.

Finalmente, cabe señalar que, a nuestro entender, el estudio de las CCAA españolas puede servir de referencia en otros contextos por varios motivos. Entre otros aspectos, el proceso de descentralización realizado en España hacia las CCAA tenía entre sus objetivos el de mejorar la rendición de cuentas del Sector Público, acercando las administraciones al ciudadano. Por tanto, el análisis de la difusión de información sobre sostenibilidad puede ayudar a tomar decisiones sobre posibles reformas administrativas. Por otra parte, la identificación de posibles factores explicativos de las políticas de difusión de información sobre sostenibilidad, en tanto que el contexto sociopolítico español es similar al de otros países de su entorno, puede tomarse como referencia de cara a la implementación de medidas de divulgación de información.

\section{Metodología de investigación}

Las CCAA españolas, al no estar obligadas, no publican informes sobre sostenibilidad formalmente presentados. No obstante, nuestro trabajo parte de la concepción de que la ausencia formal de informes de sostenibilidad no significa que las entidades públicas no estén interesadas en el tema o que no publiquen esta información a través de otras vías (Frost, 2007).

De entre todos los medios de difusión posibles, para los fines de este trabajo, hemos analizado la publicación realizada por los gobiernos regionales a través de Internet. Como señalan Muñoz y Chain (2004), el uso que las administraciones públicas hacen de Internet como herramienta de difusión de información pública ha supuesto un importante avance en el servicio a los ciudadanos. Para Gandía y Archidona (2008), Internet ha contribuido a mejorar la disponibilidad de información de las administraciones públicas. Gallego-Álvarez et al. (2010) indican que el uso de Internet ha ayudado a romper las barreras entre los ciudadanos y las administraciones públicas, proporcionando más información y con mayor frecuencia.

En este sentido, el e-gobierno ha supuesto un impulso hacia la divulgación de información gubernamental. Así, autores como Hodges y Grubnic (2010) señalan que el e-gobierno facilita, a través de Internet, el acceso público a la información sobre los servicios ofertados, permitiendo realizar transacciones. Así, la implantación del e-gobierno puede promover prácticas de 
sostenibilidad mediante la mejora de los servicios públicos, la administración pública y la interacción entre el gobierno y los ciudadanos (Estevez y Janowski, 2013).

En esta línea, el e-gobierno establece las bases para planteamientos más colaborativos entre gobernantes y ciudadanos. La introducción del e-gobierno ha supuesto un importante progreso en las relaciones de los gobiernos con los distintos stakeholders, promoviendo y facilitando la transparencia y la rendición de cuentas (Bertot et al., 2010). Además, autores como Greco et al. (2012) y Bryson et al. (2014) afirman que los procesos de implementación del e-gobierno están otorgando a los ciudadanos un nuevo papel en sus relaciones con los gobiernos, pasando de meros espectadores, a participantes en la resolución de problemas. Más aún, estos autores argumentan que la participación de los ciudadanos en las actividades de los gobiernos podría influir positivamente en la transparencia. De este modo, la implantación del e-gobierno favorece el desarrollo de una concepción más amplia, que, bajo el título de open-government, establece como principios de la gestión pública la transparencia, la colaboración y la participación (Calderón y Lorenzo, 2010).

En el ámbito de los informes de sostenibilidad, como hemos resaltado en el epígrafe anterior, las guías emitidas por el GRI son las principales referencias a nivel internacional (Lodhia et al., 2012). Por ello, nuestro trabajo considera los indicadores propuestos en la G3 y los suplementos para las entidades públicas (GRI, 2005, 2006, 2010). Esta opción supone, además, adoptar la postura del triple-bottom-line de Elkington (1998). Aunque podríamos contemplar el uso de otras guías (OCDE, 2006; Banco Mundial, 2008; AccountAbility, 2008a y 2008b; Pacto Mundial de Naciones Unidas, 2009), creemos que al centrarnos en la propuesta de las guías del GRI los resultados empíricos son más objetivos, imparciales y neutrales que si decidiéramos seleccionar ítems de distintas guías.

Así, analizamos la difusión de información usando un listado ${ }^{2}$ de 61 ítems divididos en siete apartados, que podemos agrupar en cuatro bloques: a) información general (ítems 1 a 25), que abarca información sobre estrategia, perfil de la organización, características de la información divulgada y gobierno, compromisos y participación de grupos de interés; $b$ ) información económica (ítems 26 a 41); c) información social (ítems 42 a 48); d) información medioambiental (ítems 49 a 61).

2 Al final del presente artículo, en el Anexo, se encuentra dicho listado detallado individualmente (Nota de los editores). 
Con base en trabajos previos sobre e-gobierno (Rodríguez et al., 2007), analizaremos las webs (tanto la principal como las de los distintos departamentos) de las 17 CCAA, valorando la publicación (valor 1) o no (valor 0) de cada ítem de nuestro cuestionario, de forma que podemos hacer una valoración tanto de la difusión de un determinado ítem (suma de las observaciones en las distintas CCAA) como de la difusión global realizada por una determinada CCAA (suma de las observaciones en los distintos ítems para una CCAA concreta). El análisis de las páginas web se llevó a cabo durante el primer trimestre de 2014.

En este trabajo pretendemos evaluar el nivel de información divulgada en función de los criterios establecidos por la propuesta del GRI, por ello la evaluación realizada ha sido coherente con ese fin. En este sentido, los aspectos incluidos en el listado que evaluamos están referidos a la difusión o no de información, por lo que hemos evaluado positivamente la presencia en la web de cualquier información relacionada con un ítem concreto.

Finalmente, considerando los objetivos de este trabajo, y con base en las recomendaciones del Eurostat (2012), la literatura previa citada en páginas anteriores, y la teoría de la legitimidad y la teoría de los stakeholders, creemos de interés comparar la situación observada en cuanto a difusión de información sobre sostenibilidad con datos sobre, por un lado, la situación financiera, y por otro, los compromisos de transparencia y rendición de cuentas.

Para el análisis de la situación financiera de las CCAA disponemos de los datos oficiales de deuda y déficit publicados por IGAE. En cuanto a la segunda comparativa, utilizaremos los resultados publicados por Transparencia Internacional España (TIE, 2013), ONG que trabaja en la promoción de la transparencia y la rendición de cuentas, evaluando distintas instituciones públicas, entre las que se encuentran los Gobiernos Regionales.

Además, mediante un análisis de correlación de rangos de Spearman (técnica para casos y muestras similares en numerosos estudios como la mejor herramienta estadística para testar variables que siguen un rango ordenado) (Stevenson, 1981; Sánchez, 2004; Rodríguez y Navarro, 2007), vamos a comparar los niveles de divulgación obtenidos en nuestro análisis (tanto divulgación total, como divulgación para cada uno de los bloques de nuestro cuestionario), por un lado, con los niveles de deuda y déficit per cápita, y por otro, con los resultados obtenidos por el ranking elaborado por TIE. 


\section{Resultados}

En la Tabla $1^{3}$ se reúnen los datos obtenidos para cada CCAA en cada uno de los bloques analizados. A partir de estos datos, observamos que las CCAA españolas, en su conjunto, difunden un $61.23 \%$ de la información del listado de ítem del GRI, si bien hay una importante variabilidad entre el gobierno que más difunde, con un $75.41 \%$ (Madrid), y el que menos, con un $49.18 \%$ (Cantabria), siendo ésta la única CCAA que se encuentra por debajo del $50 \%$ de divulgación.

Si observamos el conjunto de las CCAA, el Bloque III, información social (ítems 42 a 48), es el que presenta un nivel de respuesta más alto (82.35\%), aunque con menor heterogeneidad. El Bloque I (ítems 1 a 25) muestra un nivel de difusión más reducido (55.05\%). El Bloque IV, información medioambiental (ítems 49 a 61), es el único que presenta una CCAA (Cataluña) que difunde todos los ítems incluidos en el listado, si bien es al mismo tiempo el de mayor dispersión, con un nivel mínimo de 30.76\% (Extremadura y Castilla La Mancha). Llama la atención que el Bloque II, aspectos económicos (ítems 26 a 41), sobre el que tradicionalmente se ha centrado la información difundida por las entidades públicas en España, exhiba un nivel de difusión medio del $62.23 \%$, especialmente si observamos el valor mínimo, $31.25 \%$ (Galicia).

Analizando ahora los ítems divulgados, podemos ver que 20 de los 61 ítems son difundidos en las webs de todas las CCAA, además de otros cuatro que son difundidos por 16 de los 17 casos analizados. Por tanto, casi el $40 \%$ de la información está presente en las webs de las CCAA españolas. En el lado opuesto, algo más del $20 \%$ de los aspectos analizados no son incluidos en las webs de las CCAA, únicamente ocho de los 61 ítems no son difundidos en ninguna de las webs, y en otros cinco casos, sólo uno de los 17 casos estudiados ha difundido la información.

Entre aquellos ítems que han sido difundidos por todas las CCAA, casi la mitad (9) corresponden al Bloque I (ítems 1 a 25). En concreto, se trata de datos sobre la propia organización (organización, responsables, localización, etc.) o sobre la caracterización de la información (fecha de publicación, periodo que abarca, frecuencia de presentación, etc.). Otros cuatro ítems divulgados por todas las CCAA corresponden a información económica (Bloque II, ítems 26 a 41), en particular, datos presupuestarios sobre el conjunto de la entidad.

3 Todas las tablas se encuentran en el Anexo, al final del presente artículo (Nota de los editores). 
En el Bloque III (ítems 42 a 48), sobre cuestiones sociales, cinco de los siete ítems considerados en el listado están disponibles en las webs de las 17 CCAA. Finalmente, sólo dos de los 13 ítems sobre aspectos medioambientales (ítems 49 a 61) son difundidos en todas las webs analizadas. En concreto, uno referido a las inversiones medioambientales realizadas y otro sobre la promoción de productos o servicios con ahorro energético. En este apartado también encontramos dos de los cuatro ítems difundidos por 16 de las 17 CCAA (información relativa a iniciativas destinadas al ahorro energético).

Entre los ítems no difundidos por ninguno de las CCAA, la mitad (4) corresponde al Bloque I (ítems 1 a 25), en particular no se informa sobre desafíos y metas a medio plazo, perspectivas de desempeño respecto de los objetivos, prioridades en materia medioambiental, ni cuáles son los criterios para seleccionar los grupos de interés. En el Bloque II (ítems 26 a 41) son tres los ítems no difundidos por ninguna CCAA, los gastos por tipo de pago, la relación del salario de nuevos contratos respecto al salario mínimo y el gasto en proveedores locales respecto del gasto total.

En el Bloque III (ítems 42 a 48), sólo un ítem no es difundido por ninguna CCAA: el de obligaciones de pensiones con los empleados, lo cual puede deberse a que las entidades públicas españolas no suelen hacer coberturas adicionales a las que realiza el sistema público de pensiones. Respecto al Bloque IV (ítems 49 a 61), todos los ítems son divulgados, y sólo el relativo al nivel de reducción del impacto medioambiental logrado con las actuaciones adoptadas al efecto es difundido por una de las 17 CCAA.

Por otro lado, la Tabla 2 muestra el número y porcentaje de ítems divulgados por cada una de las CCAA, comparados tanto con los niveles de deuda y déficit per cápita, así como con la posición en el ranking TIE. Por último, la Tabla 3 exhibe los resultados del análisis de correlación de rangos de Spearman.

Como podemos observar en la Tabla 3, excepto los índices referidos a la información social y la general, el resto tiene un valor inferior a 0,3 ; por lo tanto, la relación es baja o nula. Así, la información divulgada por las CCAA presenta una relación moderada-fuerte, de carácter negativo $(r=-0.5246)$ entre el porcentaje de divulgación de información social y el índice TIE, y una relación moderada-baja, también de carácter negativo $(r=-0.3443)$ entre la divulgación de información general y el déficit per cápita. Por otra parte, la deuda per cápita no parece ejercer influencia en el nivel de divulgación de información sobre sostenibilidad por parte de las CCAA. 


\section{Síntesis y conclusiones}

La difusión de información sobre sostenibilidad por parte de las entidades públicas ha sido escasa hasta el momento. Entre las entidades que sí están emitiendo informes de sostenibilidad, las guías del GRI son las más usadas, motivo por el cual hemos decidido utilizarlas como base de nuestro estudio.

Asimismo, para los objetivos del trabajo, optamos por la elección de los gobiernos regionales en España, justificando la elección en varios motivos, cuya base está en la búsqueda de legitimidad en el marco de la crisis de las finanzas públicas. Además, estos gobiernos regionales, en un contexto legal común, que no exige la publicación de informes de sostenibilidad, y ante la diversidad en su contexto sociopolítico y económico, nos permiten hacernos una idea respecto de las prioridades acerca de la información sobre sostenibilidad divulgada.

A la vista del primer objetivo, es decir, si se divulga o no información sobre sostenibilidad y, en caso afirmativo, de qué tipo, la repuesta es que, aunque no se difunden formalmente informes de sostenibilidad, la mayor parte de la información necesaria para estos informes no sólo está disponible, sino que además ya es difundida en las webs de las CCAA españolas. Por tanto, podemos confirmar los planteamientos de Frost (2007), quien afirmaba que no emitir informes no implica que las entidades no estén interesadas o que no se divulgue esa información por otros medios, en nuestro caso Internet. Por otra parte, también hemos de reconocer que dicha información está dispersa y su difusión no se realiza de forma coordinada, por lo que un usuario interesado tendría dificultades para disponer de una visión de conjunto.

Además, si bien la información difundida es heterogénea, hemos de remarcar que casi el $40 \%$ de los ítems incluidos en la guía GRI ha sido difundido en por lo menos 16 de las 17 CCAA, y más del 60\% es difundido por alguna de las entidades analizadas. Por otra parte, sólo el 13\% de los ítems no ha sido divulgado por ninguna de las CCAA y aproximadamente un $8 \%$ solamente lo ha sido por una entidad. Entre los ítems sin respuesta o difundidos por una única entidad encontramos, fundamentalmente, aquellos que tienen que ver con el análisis y estrategia de la entidad o el análisis de stakeholders, así como algunos apartados económicos.

Por el contrario, entre los ítems difundidos por todas o casi todas las CCAA resalta un número importante de cuestiones económicas y, sobre todo, la difusión de los ítems de tipo social. A la vista de estos resultados, la divulgación de información parece responder a las necesidades de legitimar las actuaciones, en línea con la teoría de la legitimidad, más que en dar respuesta a los intereses de los distintos grupos de stakeholders. 
En cuanto al tipo de información publicada, un análisis detenido de los ítems divulgados por las CCAA permite obtener interesantes conclusiones sobre la difusión de información de sostenibilidad. En los entes estudiados, respecto de estos dos primeros bloques (información general y económica), hemos de resaltar que se trata de información tradicionalmente exigida a las entidades públicas. Ello explica el importante número de ítems divulgados en todas las CCAA.

No obstante, en conjunto, el nivel de difusión en las webs no es muy alto. En particular, es llamativo que el bloque económico no presente un nivel más alto de divulgación de información, toda vez que es el que cuenta con mejores y más desarrollados sistemas de información, ya que sí existe un requerimiento legal para la divulgación de los principales aspectos económico-presupuestarios. En cualquier caso, sí corroboramos que la información exigida legalmente es difundida en todos los casos, siendo los ítems que relacionan aspectos económicos con otros, como el personal o los proveedores, los que no se difunden.

Respecto al Bloque I, de información general, los aspectos sobre los que no se difunde información se concentran en los relativos a estrategias y análisis sobre la sostenibilidad de la entidad, en particular la declaración del máximo responsable sobre la relevancia de la sostenibilidad en la estrategia de la entidad, en cuanto a prioridades medioambientales o a selección de los grupos de interés.

Dentro de los aspectos divulgados, y siguiendo los ítems del GRI, resalta la difusión de información social. Esta circunstancia podría explicarse, desde la perspectiva de la teoría de los stakeholders, sugiriendo que las CCAA están más sensibilizadas hacia las demandas de los grupos de interés con niveles adquisitivos más bajos.

A la vista de estos resultados podemos afirmar que las entidades públicas cuentan con información suficiente como para abordar la elaboración de informes de sostenibilidad en línea con las exigencias de las guías GRI. En este sentido, creemos que la publicación de informes de sostenibilidad, además de permitir a la organización reflexionar sobre su situación y motivar acciones futuras (Bebbington et al., 2008), redundarían en la importancia que las entidades públicas conceden a la sostenibilidad y mejorarían la transparencia de las entidades públicas, colaborando legitimar sus actuaciones ante la ciudadanía.

No obstante, la posible emisión de estos informes precisa, además de una labor de coordinación y recopilación en el conjunto de la entidad, de una concienciación por parte de los principales responsables de estas entidades públicas. Esto es así, como muestran los resultados, porque precisamente la 
información sobre planificación y estratégica de la organización, así como los datos sobre stakeholders, son los menos publicados en las web.

En cuanto a los posibles factores influyentes, y teniendo en cuenta que el Fiscal Sustainability Report 2012 de la UE (2012) señala que los niveles actuales de deuda y déficit pueden estar influyendo y resultando perjudiciales para la sostenibilidad, nuestros resultados muestran que, en general, ambas magnitudes no tienen incidencia en la divulgación de información sobre sostenibilidad de las CCAA (excepto la relación moderada-baja, y de carácter negativo, entre el déficit y la información genérica).

A pesar de la mala situación financiera de las CCAA, los niveles de deuda y déficit per cápita no tienen influencia en la divulgación de información sobre sostenibilidad, por lo que éstos no están impulsando de manera positiva a los gobiernos para divulgar información sobre sostenibilidad. Por ende, desde la perspectiva de la teoría de la legitimidad, la situación financiera no parece ser un aspecto clave que explique la preocupación por la sostenibilidad en las CCAA.

Por otra parte, según los resultados de nuestro análisis, la transparencia general en la información sobre sostenibilidad podría tener un impacto negativo en la transparencia de la información social. Por lo tanto, realizar campañas de comunicación en las diferentes áreas/departamentos dentro de las CCAA, como parte de la política general de la institución en materia de transparencia, con el objetivo de incrementar la conciencia entre los administradores de la importancia de publicar información acerca de la sostenibilidad, podría ser contraproducente en lo que a la divulgación de información sobre sostenibilidad se refiere.

Como aspectos a tomar en cuenta en el futuro, estarían (además de los ya señalados y relativos a una revisión de los ítems considerados a partir de otras guías), por un lado, el análisis de variables contextuales que pudiesen afectar a los resultados obtenidos. En este sentido, las CCAA españolas presentan ventajas, ya que permite el análisis de variables sociopolíticas y económicas manteniendo otras constantes, pero también inconvenientes, ya que, aunque trabajamos con la población completa, el número de observaciones es muy reducido, lo cual dificulta los análisis estadísticos. Por otro lado, retomando a Marcuccio y Steccolini (2005), sería interesante profundizar en las razones de la difusión de la información de sostenibilidad, en el motivo de por qué se centra en unos aspectos y no en otros. 
Francisco J. Alcaraz-Quiles, Andrés Navarro-Galera y David Ortiz-Rodríguez. La transparencia sobre sostenibilidad en gobiernos regionales: el caso de España

\section{Referencias}

AccountAbility (2008a), AA1000 AccountAbility Principles Standard, Inglaterra: Accountability.

AccountAbility (2008b), AA1000 Assurance Standard 2008, Inglaterra: Accountability.

Ball, Amanda (2004), "A Sustainability Accounting Project for the UK Local Government Sector? Testing the Social Theory Mapping Process and Locating a Frame of Reference", en Critical Perspectives on Accounting, núm. 15, Inglaterra: Elsevier on-line. Disponible en: http://www.sciencedirect.com/science/article/pii/S1045235402002095 [1 de octubre de 2015].

Banco Mundial (2008), "Overview: Understanding, Measuring and Overcoming Poverty". Disponible en: http://web.worldbank.org [30 de agosto de 2013].

Banerjee, Subhabrata B. (2007), Corporate Social Responsibility: The Good, the Bad and the Ugly, Inglaterra: Edward Elgar.

Bebbington, Jan et al. (2008), "Corporate social reporting and reputation risk management", en Accounting, Auditing and Accountability Journal, núm. 21, Reino Unido: Emerald on-line. Disponible en: http://www.emeraldinsight.com/doi/ pdfplus/10.1108/09513570810863932 [1 de octubre de 2015].

Bertot, John C. et al. (2010), "Using ICTs to create a culture of transparency: E-government and social media as openness and anti-corruption tools for societies", en Government Information Quarterly, núm. 27, Inglaterra: Elsevier on-line. Disponible en: http:// www.sciencedirect.com/science/article/pii/S0740624X10000201 [1 de octubre de 2015].

Bryson, John M. et al. (2014), "Public Value Governance: moving beyond traditional public administration and the New Public Management", en Public Administration Review, núm. 74, Estados Unidos: Willey Online Library. Disponible en: http://onlinelibrary. wiley.com/doi/10.1111/puar.12238/epdf [1 de octubre de 2015].

Calderón, César y Lorenzo, Sebastián (2010), Open governmenet: Gobierno abierto, España: Algón Editores.

Canyelles, Josep M. (2011), "Responsabilidad Social de las Administraciones Públicas", en Revista de Contabilidad y Dirección, núm. 13, España: ACCID.

Coglianese, Cary (2009), " The transparency President? The Obama administration and open government", en Governance, núm. 22, Estados Unidos: John Wiley \& Sons online. Disponible en: https://papers.ssrn.com/sol3/papers.cfm?abstract_id $=1433815$ [1 de octubre de 2015].

Contreras, Leticia (2010), "La calidad en la gestión como factor de cambio institucional en las organizaciones gubernamentales del Estado de México", en Convergencia. Revista de Ciencias Sociales [en línea], vol. 17, mayo-agosto, México: Universidad Autónoma del Estado de México. Disponible en: http://www.redalyc.org/articulo. oa?id=10513135012 [30 de agosto de 2013].

Crane, Andrew y Matten, Dirk (2007), Business ethics: managing corporate citizenship and sustainability in the age of globalization, Inglaterra: Oxford University Press.

Crane, Andrew et al. (2008), Corporate Social Responsibility. Readings and cases in a Global Context, Estados Unidos: Routledge.

Crognale, Gabriele (2009), “The Global Reporting Initiative G3 Sustainability Reporting Guidelines (Conclusion)", en Business and the Environment, núm. 20, Gran Bretaña: Wolters Kluver on-line. Disponible en: http://www.hbs.edu/environment/Pages/ default.aspx [1 de octubre de 2015]. 
Deegan, Craig (2002), “The legitimising effect of social and environmental disclosures - A theoretical foundation?", en Accounting, Auditing and Accountability Journal, núm. 15, Reino Unido: Emerald on-line. Disponible en: http://www.emeraldinsight.com/doi/ pdfplus/10.1108/09513570210435852 [1 de octubre de 2015].

Deegan, Craig y Unerman, Jeffrey (2006), Financial Accounting Theory, Australia: McGraw Hill.

Dumay, John C. et al. (2010), "GRI sustainability reporting guidelines for public and third sector organizations”, en Public Management Review, núm. 12, Gran Bretaña: Taylor \& Francis on-line. Disponible en: http://www.tandfonline.com/doi/full/10.1080/1471 9037.2010.496266? scroll=top\&needAccess=true [ 1 de octubre de 2015].

Elkington, John (1998), Cannibals with Forks. The Triple Bottom Line of the 21st Century, Reino Unido: Capstone Publishing.

Erdmenger, Christoph (1998), "From business to municipality-and back", en Local Environment, núm. 3, Gran Bretaña: Taylor \& Francis on-line. Disponible en: http:// www.tandfonline.com/doi/abs/10.1080/13549839808725573?journalCode $=\mathrm{cl}$ oe20[1 de octubre de 2015].

Estevez, Elsa y Janowski, Tomasz (2013), "Electronic Governance for Sustainable Development-Conceptual framework and state of research", en Government Information Quarterly, núm. 30, Estados Unidos: Elsevier on-line. Disponible en: http://www.sciencedirect.com/science/article/pii/S0740624X12001487 $[1$ de octubre de 2015].

Eurostat (2012), Eurostat Newrelease Euroindicators 149/2012. Disponible en: http://epp. eurostat.ec.europa.eu/cache/ITY_PUBLIC/2-22102012-AP/EN/2-22102012-APEN.PDF [30 de agosto de 2013].

Farneti, Federica y Guthrie, James (2009), "Sustainability Reporting by Australian Public Sector Organizations”, en Accounting forum, núm. 33, Australia: Elsevier on-line. Disponible en: http://www.sciencedirect.com/science/article/pii/ S015599820900009X [1 de octubre de 2015].

Fox, Tom et al. (2002), Public sector roles in strengthening corporate social responsibility: a baseline study, Inglaterra: International Institute for Environment and Development.

Frost, Geoff (2007), "The introduction of mandatory environmental reporting guidelines: Australian Evidence”, en Abacus, núm. 43, Estados Unidos: John Wiley \& Sons online. Disponible en: https://papers.ssrn.com/sol3/papers.cfm?abstract_id=988569 [1 de octubre de 2015].

Gallego-Álvarez, Isabel et al. (2010), "Are determining factors of municipal E-government common to a worldwide municipal view? An intra-country comparison", en Government Information Quarterly, núm. 27, Estados Unidos: Elsevier on-line. Disponible en: http://www.sciencedirect.com/science/article/pii/S0740624X10000596 $[1$ de octubre de 2015].

Gandía, Juan L. y Archidona, María. C. (2008), "Determinants of web site information by Spanish city councils", en Online Information Review, núm. 32, Reino Unido: Emerald on-line. Disponible en http://www.emeraldinsight.com/doi/ full/10.1108/14684520810865976 [1 de octubre de 2015].

Gil-Ruiz, Carmen L. e Iglesias, Jaime (2007), "El gasto público en España en un contexto descentralizado”, en Presupuesto y Gasto Público, núm. 47, España: Instituto de Estudios Fiscales.

GRI (Global Reporting Initiative) (2005), Sector Supplement for Public Agencies, Holanda: GRI. 
Francisco J. Alcaraz-Quiles, Andrés Navarro-Galera y David Ortiz-Rodríguez. La transparencia sobre sostenibilidad en gobiernos regionales: el caso de España

GRI (2006), G3 Sustainability Reporting Guidelines, Holanda: GRI.

GRI (2010), Reporting in Government Agencies, Holanda: GRI.

Goodpaster, Kenneth E. (2009), Ética empresarial y análisis de los “stakeholders", España: Aurkilan Spanish Annual.

Gray, Rob (2006), "Social, environmental and sustainability reporting and organisational value creation: whose value? Whose creation?", en Accounting, Auditing and Accountability Journal, núm. 19, Reino Unido: Emerald on-line. Disponible en: http://www.emeraldinsight.com/doi/full/10.1108/09513570610709872 $\left[\begin{array}{ll}1 & \text { de }\end{array}\right.$ octubre de 2015].

Greco, Giulio et al. (2012), "From Tuscany to Victoria: Some Determinants of Sustainability Reporting by Local Councils", en Local Government Studies, núm. 38, Gran Bretaña: Taylor \& Francis on line. Disponible en: http://www.tandfonline.com/doi/abs/10.10 80/03003930.2012.679932 [1 de octubre de 2015].

Grupo Banco Mundial (2007), Environmental, Health, and Safety (EHS) Guidelines, Estados Unidos: Environment and Social Development Department, International Finance Corporation.

Guthrie, James et al. (2010), "Advancing sustainable management of public and not for profit organizations”, en Public Management Review, núm. 12, Gran Bretaña: Taylor \& Francis on-line. Disponible en: http://www.tandfonline.com/doi/abs/10.1080/1471 9037.2010.496254 [1 de octubre de 2015].

G-20 (2013), G-20 Leaders' Declaration. Document, St. Petesburgo, Rusia, 6 de Septiembre de 2013. Disponible en: www.g20.org [30 de octubre de 2013].

Herbohn, Kathleen (2005), "A Full Cost Environmental Accounting Experiment", en AccountingOrganisation and Society, núm.30, Gran Bretaña:Elsevieron-line.Disponible en: http://www.sciencedirect.com/science/article/pii/S0361368205000024 [1 de octubre de 2015.]

Hodges, Ron y Grubnic, Suzana (2010), "Local authority e-government partnerships in England: a case study”, en Financial Accountability and Management, núm. 26, Gran Bretaña: John Wiley \& Sons on-line. Disponible en: http://onlinelibrary.wiley.com/ doi/10.1111/j.1468-0408.2009.00490.x/pdf [1 de octubre de 2015].

IFAC (2013), Reporting on the Long-Term Sustainability of an Entity's Finances. Disponible en: http://www.ifac.org/publications-resources/recommended-practice-guideline-1 [30 de agosto de 2014].

IGAE (Intervención General de la Administración del Estado) (2015), Avance actuación económica y financiera de las Administraciones Públicas, España: IGAE. Disponible en http://www.igae.pap.meh.es [10 de enero de 2016].

International Federation of Accountants (IFAC) (2011), Illuminating the Issues Highlighting the Solutions. Disponible en: http://viewer.zmags.com/publication/ed4395af\#/ ed4395af/1_[30 de agosto de 2013].

Jeffery, Charlie (1997), The regional dimension of the European Union: Towards a third level in Europe?, Inglaterra: Frank Cass.

Jordana, Jacint et al. (2005), "Which Internet Policy? Assessing Regional Initiatives in Spain”, en The Information Society, núm. 21, Gran Bretaña: Taylor \& Francis on-line. Disponible en: http://www.tandfonline.com/doi/abs/10.1080/01972240500253509 [1 de octubre de 2015]. 
Lansu, Angelique et al. (2013), "Changing professional demands in sustainable regional development: a curriculum design process to meet transboundary competence", en Journal of Cleaner Production, núm. 49, Estados Unidos: Elsevier on-line. Disponible en: http://www.sciencedirect.com/science/article/pii/S0959652612005513 [1 de octubre de 2015].

Lamprinidi, Stefania y Kubo, Naoko (2008), “Debate: The Global Reporting Initiative and Public Agencies”, en Public Money \& Management, núm. 28, Gran Bretaña: Taylor \& Francis on-line. Disponible en: http://www.tandfonline.com/doi/abs/10.1111/ j.1467-9302.2008.00663.x [1 de octubre de 2015].

Le Galès, Patrick y Lequesne, Christian (1998), Regions in Europe, Inglaterra: Routledge.

Lodhia, Sumit et al. (2012), "Driving Public Sector Environmental Reporting”, en Public Management Review, núm. 14, Gran Bretaña: Taylor \& Francis on-line. Disponible en: http://www.tandfonline.com/doi/abs/10.1080/14719037.2011.642565 [1 de octubre de 2015].

Marcuccio, Manila y Steccolini, Iliana (2005), "Social and environmental reporting in local authorities", en Public Management Review, núm. 7, Gran Bretaña: Taylor \& Francis on-line. Disponible en: http://www.tandfonline.com/doi/ abs/10.1080/14719030500090444 [1 de octubre de 2015].

Martinov-Bennie, Nonna y Hecimovic, Angela (2010), "Assurance of Australian Natural Resource Management", en Public Management Review, núm. 12, Gran Bretaña: Taylor \& Francis on-line. Disponible en: http://www.tandfonline.com/doi/abs/10.10 80/14719037.2010.496267 [1 de octubre de 2015].

Muñoz Cañavate, Antonio y Chain Navarro, Celia (2004), “The World Wide Web as an information system in Spain's regional administrations (1997-2000)”, en Government Information Quarterly, núm. 21, Estados Unidos: Elsevier on-line. Disponible en: http://www.sciencedirect.com/science/article/pii/S0740624X0400005X $[1$ de octubre de 2015].

Mussari, Riccardo y Monfardini, Patrizio (2010), "Practices of social reporting in public sector and non-profit organizations”, en Public Management Review, núm. 12, Gran Bretaña: Taylor \& Francis on-line. Disponible en: http://www.tandfonline.com/doi/ abs/10.1080/14719037.2010.496262 [1 de octubre de 2015].

Neu, Dean et al. (1998), "Managing Public Impressions: Environmental Disclosures in Annual Reports”, en Accounting, Organisations and Society, núm. 23, Gran Bretaña: Elsevier on-line. Disponible en: http://www.sciencedirect.com/science/article/pii/ S0361368297000081 [ 1 de octubre de 2015].

Navarro-Galera, Andrés et al. (2010), "La Divulgación de Información sobre Responsabilidad Social Corporativa en Administraciones Públicas: Un Estudio Empírico en Gobiernos Locales", en Revista Española de Contabilidad RC-SAR, núm. 13, España: Elsevier on-line. Disponible en: http://www.sciencedirect.com/science/ article/pii/S1138489110700194 [1 de octubre de 2015].

OCDE (Organización para la Cooperación y el Desarrollo Económicos) (2006), Intellectual Assets and Value Creation: Implications for Corporate Reporting, Francia: Corporate Affairs Division, Directorate for Financial and Enterprise Affairs, OCDE.

Orden EHA/1037/2010 (2010, 28 de abril), Leyes de España, en Boletín Oficial del Estado, núm. 102. Disponible en: http://www.boe.es/boe/dias/2010/04/28/pdfs/ BOE-A-2010-6710.pdf [30 de agosto de 2013]. 
Osborne, Stephen P., y Ball, Amanda (2011), Social Accounting and Public Management: Accountability for the Public Good, Reino Unido: Routledge.

Pacto Mundial de Naciones Unidas (2009), "What Is the Global Compact”. Disponible en: http://www.unglobalcompact.org/ [30 de agosto de 2013].

Pina, Vicente et al. (2010), "Is E-Government Promoting Convergence Towards More Accountable Local Governments?”, en International Public Management Journal, núm. 13, Estados Unidos: Taylor \& Francis on-line. Disponible en http://www.tandfonline. com/doi/abs/10.1080/10967494.2010.524834 [1 de octubre de 2015].

Potts, Tavis (2010), “The natural advantage of regions: linking sustainability, innovation, and regional development in Australia", en Journal of Cleaner Production, núm. 18, Estados Unidos: Elsevier on-line. Disponible en: http://www.sciencedirect.com/ science/article/pii/S0959652610000193 [1 de octubre de 2015].

Rodríguez, Manuel et al. (2007), "E-Government and public financial reporting: the case of Spanish regional governments", en American Review of Public Administration, núm. 37, Estados Unidos: Sage on-line. Disponible en: http://journals.sagepub.com/doi/ abs/10.1177/0275074006293193 [1 de octubre de 2015].

Rodríguez Bolivar, Manuel P. y Navarro Galera, Andrés (2007), “Could fair value accounting be useful, under NPM models, for users of financial information?”, en International Review of Administrative Sciences, núm. 73, Gran Bretaña: Sage on-line.

Rodríguez Bolivar, Manuel P. y Navarro Galera, Andrés (2007), "Could fair value accounting be useful, under NPM models, for users of financial information?”, en International Review of Administrative Sciences, núm. 73, Gran Bretaña: Sage on-line. Disponible en: http://journals.sagepub.com/doi/abs/10.1177/0020852307081153 [1 de octubre de 2015].

Ruiz-Huerta Carbonell, Jesús y García Díaz, Miguel A. (2012), "El endeudamiento de las Comunidades Autónomas: Límites y Problemas en el contexto de la crisis económica”, en Revista de Estudios Autonómicos y Federales, núm. 15, España: Instituto de Estudios Autonómicos.

Sánchez, Jesús (2004), Introducción a la Estadistica Empresarial. Disponible en: http:// www.eumed.net/cursecon/libreria/index.htm [30 de agosto de 2013].

Stevenson, William (1981) Estadistica para Administración y Economia: Conceptos y aplicaciones, México D.F.: Harla.

TIE (Transparencia Internacional España) (2013), Indice de Transparencia de las Comunidades Autónomas. Disponible en: http://www.transparencia.org.es/ [30 de agosto de 2014].

Tort, Laura E. (2010), GRI Reporting in Government Agencies, Holanda: Global Reporting Initiative (GRI).

UE (Unión Europea) (2007), Corporate Social Responsibility National public policies in the European Union, Luxemburgo: European Commission.

UE (2011), Communication from the Commission to the European Parliament, the Council, the European Economic and Social Committee and the Committee of the Regions, Bélgica: EU.

UE (2012), Fiscal Sustainability Report 2012. Disponible en: http://ec.europa.eu/ economy_finance/publications/european_economy/2012/pdf/ee-2012-8_en.pdf [30 de agosto de 2013]. 
UE (2013), Corporate Social Responsibility: accountable, transparent and responsible business behaviour and sustainable growth. Disponible en: http://www.europarl. europa.eu/oeil/popups/ficheprocedure.do? reference=2012/2098(INI)\&l=EN [30 de agosto de 2013].

United States Agency for International Development (USAID) (2011), Fundamentals of NGO Financial Sustainability, Estados Unidos: Abt Associates Inc.

Uvalle-Berrones, Ricardo (2012), "La administración pública en los imperativos de la gobernanza democrática”, en Convergencia. Revista de Ciencias Sociales [en línea], septiembre-diciembre, México: Universidad Autónoma del Estado de México. Disponible en: http://www.redalyc.org/articulo.oa?id=10522923004> ISSN 14051435 [30 de agosto de 2013].

\section{Anexo}

\section{Listado de ítems}

\section{Bloque 1. Información General}

\section{a. Estrategia y análisis:}

1. ¿Existe declaración del máximo responsable acerca de la relevancia de la sostenibilidad para la organización y para su estrategia?

2. ¿Esta declaración incluye prioridades, estrategias y asuntos clave a medio/corto plazo?

3. ¿Esta declaración incluye tendencias de mayor alcance que afectan a prioridades en materia de sostenibilidad?

4. ¿Esta declaración incluye eventos, logros y fracasos registrados durante el periodo de información?

5. ¿Esta declaración incluye perspectivas de desempeño en relación con los objetivos?

6. ¿Esta declaración incluye desafíos y metas para el próximo año y los 3-5 siguientes?

b. Perfil de la organización:

7. ¿La entidad tiene marcas propias?

8. ¿Existen distintas áreas claramente definidas?

9. ¿Existen responsables definidos por área?

10. Localización de la sede principal de la entidad.

11 Número de países en los que desarrolla actividades significativas.

12. Número de empleados.

13. ¿Se han producido cambios relevantes en la estructura o tamaño de la entidad?

14. ¿Ha obtenido la entidad premios o distinciones en el periodo que abarca la información suministrada? 
c. Parámetros de la información:

15. Periodo que abarca la información suministrada.

16. Fecha información suministrada anterior.

17. Frecuencia de presentación de la información suministrada.

18. ¿Existe punto de contacto general para cuestiones relativas a la información suministrada?

19. ¿Incluye la información suministrada datos de interés específico para proveedores y usuarios?

20. ¿Viene asignada la prioridad de los aspectos recogidos en la información suministrada?

d. Gobierno, compromisos y participación de los grupos de interés:

21. ¿Existe persona u órgano responsable de la definición de la estrategia de la organización?

22. ¿Ostenta el máximo responsable algún otro cargo público o privado?

23. ¿Existen comités de empresa o representantes de los trabajadores?

24. ¿Se incluyen a los grupos de interés en la información suministrada?

25. ¿Se incluyen criterios de identificación y selección de los grupos de interés?

Bloque 2. Información económica

26. Presupuesto de Gastos/Población beneficiada.

27. Presupuesto de Ingresos/Población beneficiada.

28. Ingresos por transferencias de otras Administraciones públicas/ingresos totales.

29. Gastos Brutos desglosados por tipo de Pago.

30. Gastos Brutos desglosados por Clasificación financiera.

31. Gastos de capital desglosados por Clasificación financiera.

32. Estipulaciones de la política de promoción interna.

33. Acciones de formación.

34. Riesgos financieros futuros.

35. Datos sobre subvenciones recibidas.

36. Divulga el presupuesto de gastos e incluye:

37. Perspectivas a medio plazo.

38. Muestra los supuestos económicos clave: previsión crecimiento PIB, empleo, tasa de desempleo, inflación y tipos de interés.

39. Gastos debidos a programas de Asuntos Sociales.

40. Salario inicial (en las primeras contrataciones de personal)/Salario mínimo local).

41. Gasto en proveedores locales/Gasto total. 


\section{Bloque 3. Información Social}

42. ¿Es pública la oferta de servicios?

43. Convocatoria de Subvenciones para actividades empresariales.

44. Revela las obligaciones de pensiones con los empleados.

45 Convocatoria de Subvenciones para Asociaciones de Vecinos.

46. Ofertas de empleo público.

47. Convocatoria de subvenciones para ONG's.

48. Indicadores de eficacia y eficiencia.

\section{Bloque 4. Información Medioambiental}

49. Difunde las iniciativas llevadas a cabo para mitigar los impactos ambientales de los productos y servicios.

50. Revela el grado de reducción de ese impacto.

51. Revela el consumo directo de energía procedente de fuentes primarias.

52. Revela el consumo de energía intermedia.

53. Difunde las acciones llevadas a cabo para incrementar el ahorro de energía mediante conservación o mejoras en la eficiencia.

54. Emite información acerca de las iniciativas llevadas a cabo para promocionar productos y servicios eficientes en el consumo de energía o basados en energías renovables.

55. Se revelan las reducciones en el consumo de energía como resultado de dichas iniciativas.

56. Difunde las iniciativas llevadas a cabo para reducir el consumo indirecto de energía.

57. Se revelan las reducciones logradas con dichas iniciativas.

58. Revela las distintas fuentes de captación de agua y el volumen por fuente.

59. Informa acerca del porcentaje y el volumen total de agua reciclada y reutilizada en la comunidad.

60. Proporciona información acerca de los vertidos y destino de las aguas residuales en la comunidad.

61. Informa acerca del total y los tipos de gastos e inversiones ambientales.

Fuente: GRI. Elaboración propia. 
Francisco J. Alcaraz-Quiles, Andrés Navarro-Galera y David Ortiz-Rodríguez. La transparencia sobre sostenibilidad en gobiernos regionales: el caso de España

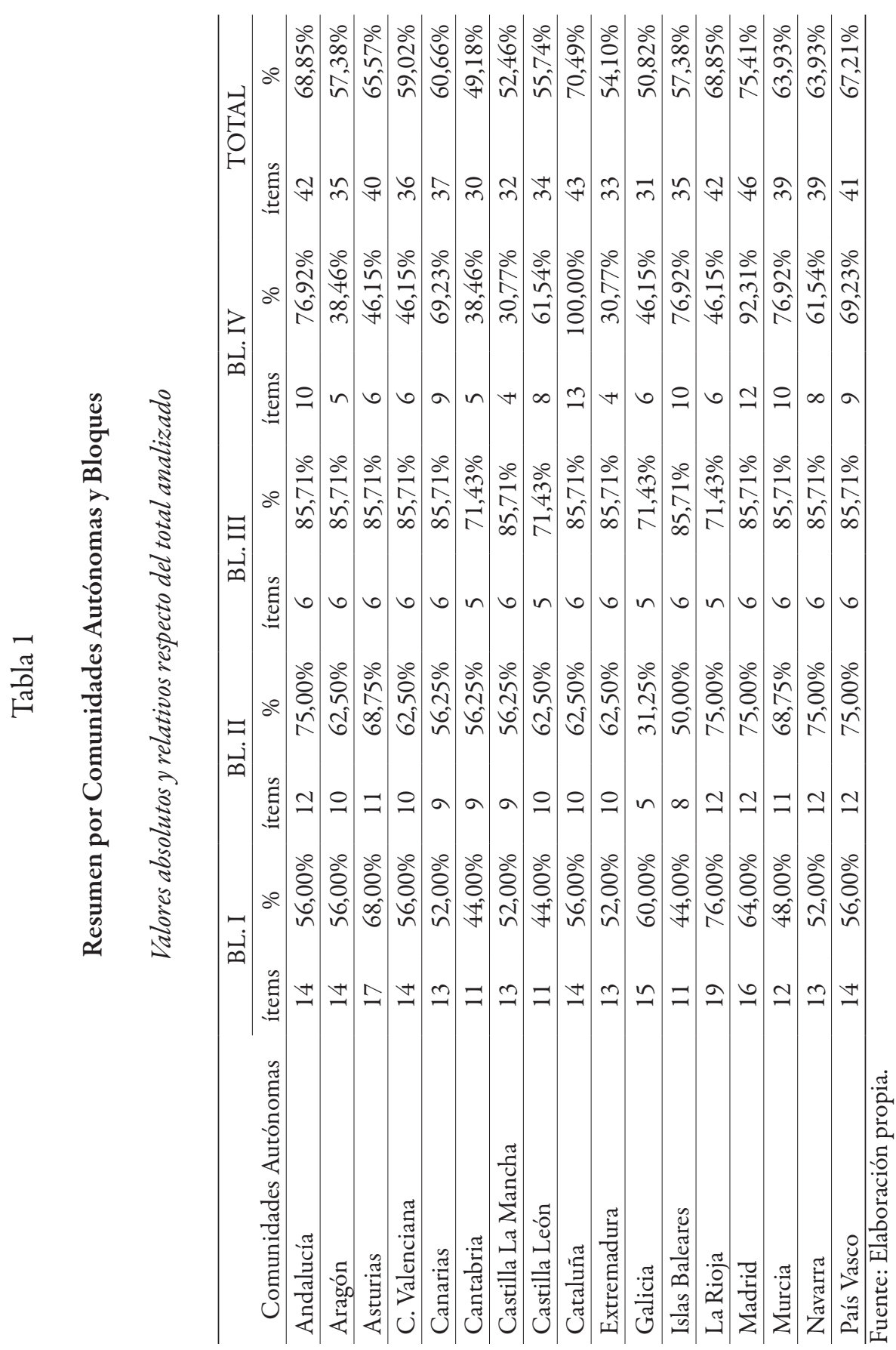




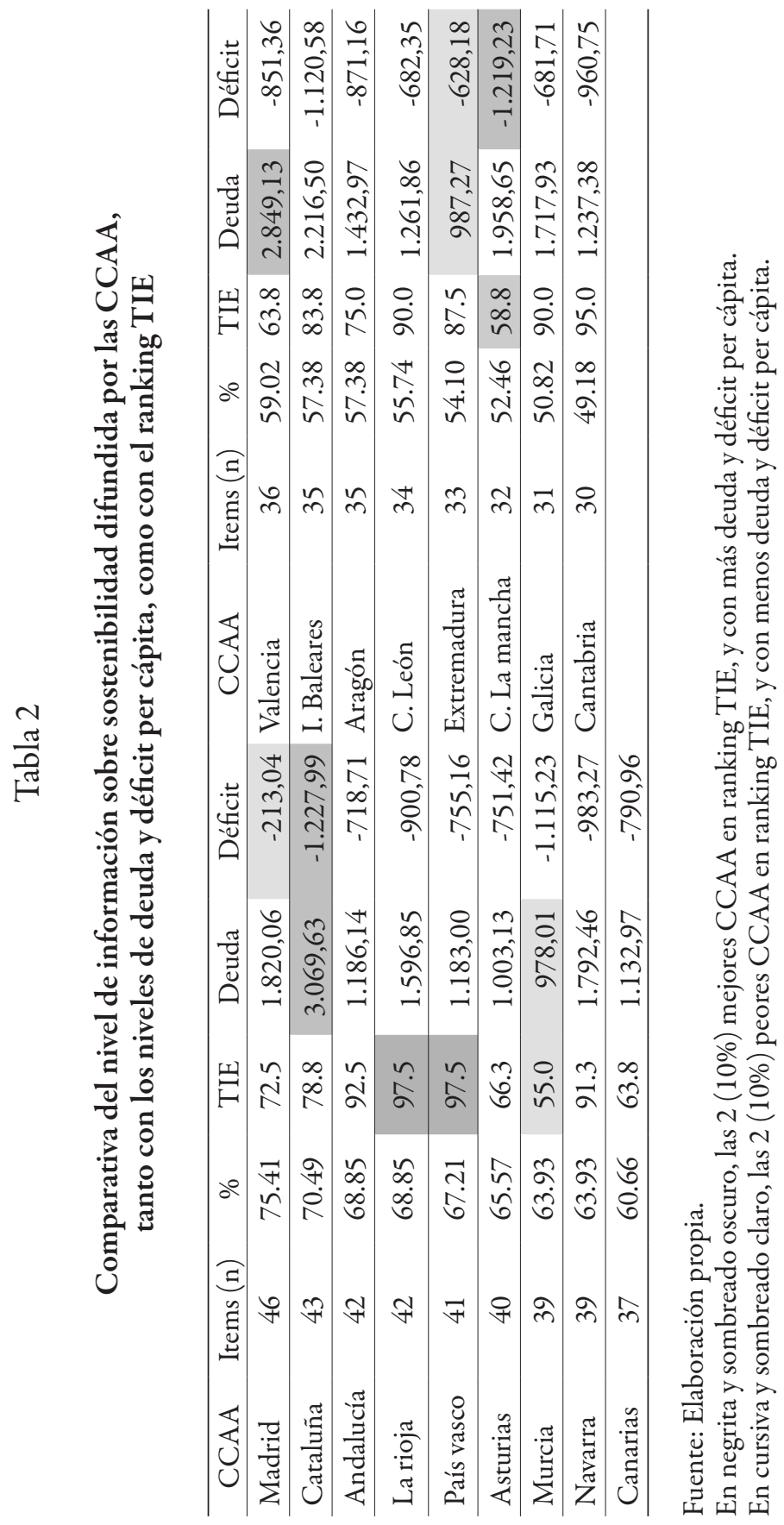


Tabla 3

Resultados del análisis de correlación de Rangos de Spearman

\begin{tabular}{lccccccccc} 
& CCAA & & CCAA & & CCAA & & CCAA & & CCAA \\
\hline TOTAL & & BL 1 & & BL 2 & & BL 3 & & BL 4 & \\
TIE & 0,0535 & TIE & 0,0847 & TIE & 0,2867 & TIE & $-0,5246$ & TIE & $-0,0411$ \\
\hline TOTAL & & BL 1 & & BL 2 & & BL 3 & & BL 4 & \\
DEB & 0,0602 & DEB & 0,1127 & DEB & $-0,2118$ & DEB & 0,0000 & DEB & 0,1411 \\
\hline TOTAL & & BL 1 & & BL 2 & & BL 3 & & BL 4 & \\
DEF & $-0,0221$ & DEF & $-0,3443$ & DEF & $-0,1992$ & DEF & 0,1698 & DEF & 0,1025 \\
\hline
\end{tabular}

Fuente: Elaboración propia.

Francisco J. Alcaraz-Quiles. Doctor. Profesor Sustituto Interino. Departamento de Economía Financiera y Contabilidad. Universidad de Granada, España. Líneas de investigación: RSC y sostenibilidad en administraciones públicas. Publicaciones recientes: "Factors determining online sustainability reporting by local governments", en International Review of Administrative Sciences, Sage (2014); "A comparative analysis of transparency in sustainability reporting by Local and Regional Governments", en Lex Localis - Journal of self-government, Maribor, Graz, Trieste, Split (2014); "Factors influencing the transparency of sustainability information in regional governments: an empirical study", en Journal of Cleaner Production, Elsevier (2014).

Andrés Navarro-Galera. Catedrático de Universidad. Departamento de Economía Financiera y Contabilidad, Universidad de Granada, España. Líneas de investigación: RSC y sostenibilidad en administraciones públicas; evaluación de la eficacia y eficiencia de servicios públicos; análisis y aplicación de normas internacionales de información financiera en administraciones públicas. Publicaciones recientes: "New development: The role of accounting in assessing local government sustainability", en Public Money and Management, Taylor and Francis (2014); "Factors determining online sustainability reporting by local governments", en International Review of Administrative Sciences, Sage (2014); "Factors influencing the transparency of sustainability information in regional governments: an empirical study", en Journal of Cleaner Production, Elsevier (2014). 
David Ortiz-Rodríguez. Profesor Titular de Universidad. Departamento de Economía Financiera y Contabilidad, Universidad de Granada, España. Líneas de investigación: RSC y sostenibilidad en administraciones públicas; evaluación de la eficacia y eficiencia de servicios públicos; análisis y aplicación de normas internacionales de información financiera en administraciones públicas. Publicaciones recientes: "Administrative Reforms to Governmental Financial Information Systems in GCC Countries: The Case of Qatar", en Middle Eastern Studies, Taylor and Francis (2013); "Factors determining online sustainability reporting by local governments", en International Review of Administrative Sciences, Sage (2014); "A comparative analysis of transparency in sustainability reporting by Local and Regional Governments", en Lex Localis-Journal of self-government, Maribor, Graz, Trieste, Split (2014).

Recepción: 11 de junio de 2015.

Aprobación: 13 de septiembre de 2016. 